\title{
Corrosion resistance of tungsten and nickel in molten eutectic mixture LiF-NaF
}

\author{
Marta Ambrová, Vladimír Danielik \\ Institute of Inorganic Chemistry, Technology and Materials, \\ Faculty of Chemical and Food Technology, Slovak University of Technology in Bratislava, \\ Radlinského 9, 812 37, Bratislava, Slovakia \\ marta.ambrova@stuba.sk
}

\begin{abstract}
Corrosion resistance of tungsten and nickel in molten eutectic LiF-NaF was investigated by measurement of polarization resistance and chronopotentiometry. Results obtained by both methods were compared by digital simulation of diffusion of corrosion products. All measurements were carried out at the temperature of $680^{\circ} \mathrm{C}$ in two types of atmospheres; in inert argon and oxidation air atmosphere. It was found that presence of air (oxygen) significantly decreases corrosion resistance of used metals. From tested materials, tungsten was more corrosion resistant material than nickel in both types of atmospheres. Obtained results were also compared with those measured in the molten eutectic LiF-NaF-KF earlier.
\end{abstract}

Keywords: corrosion, chronopotentiometry, nickel, polarization resistance, tungsten

\section{Introduction}

Besides the production of light metals and chemical or electrochemical deposition of metals, the usage of molten salts in various areas of industry becomes more and more important. The most interesting seems to be their application as a heat transfer medium in a new generation of nuclear reactors (so-called Generation IV) as well as in the spent nuclear fuel treatment. This, of course, places great emphasis on the corrosion resistance of used materials. From this point of view, investigation of the corrosion of basic metals in various molten salt media and knowledge of its mechanism is necessary not only for the development of highly corrosion resistant materials but also in term of safety during some failure.

In this work, corrosion resistance of tungsten and nickel was investigated in the molten eutectic LiF-NaF at the temperature of $680{ }^{\circ} \mathrm{C}$ in two types of atmospheres; inert argon and oxidation air atmosphere. Air conditions were tested due to safety and possible risk involved during equipment failure or poor purity of molten salts, when the molten salts could come into direct contact with air oxygen. Used eutectic mixture LiF-NaF was chosen due to its intended usage in the secondary (heat transfer) circuit of nuclear reactors operating on the base of molten salts. Also, the selected metals are considered to be the major components of highly corrosion resistant materials. Obtained results were also compared with those obtained in the molten eutectic LiF-NaF-KF earlier (Ambrová and Danielik 2012).

\section{Experimental}

\section{Methodology}

Basic principles of the methods (i.e. polarization resistance and chronopotentiometry) used for the corrosion resistance investigation have already been described earlier (Ambrová and Danielik 2012).

\section{Working procedure}

Analytical grade chemicals LiF (Suprapur, Merck, Germany), NaF (Merck, Germany) and $\mathrm{NiF}_{2}$ (Aldrich, USA) were used. They were dried in a closed furnace at the temperature of $600{ }^{\circ} \mathrm{C}$ for 4 hours before the corrosion measurements. Tungsten and nickel wires, thickness of $1 \mathrm{~mm}$ and purity of $99.99 \%$, were supplied by Aldrich, USA.

Polarization resistance and chronopotentiometric studies were performed with the use of an Autolab PGSTAT20 potentiostat/galvanostat (ECO Chemie, The Netherlands) controlled by a computer. The cell consisted of a graphite crucible which, at the same time, served as the counter electrode. Metal samples (tungsten or nickel) were used as working electrodes and $\mathrm{Ni} / \mathrm{Ni}^{2+}$ electrode as reference electrode. The contact area between metal sample and melt was determined after each experiment by measuring the immersion depth in the electrolyte. A graphite crucible containing $130 \mathrm{~g}$ of dried eutectic mixture (61.0 mol. \% LiF; $39.0 \mathrm{~mol}$ \% NaF) was placed either in a closed furnace under inert argon atmosphere, or in an open furnace that provided contact with air (oxygen). Prior to furnace inlet, argon (99.99 \%, Messer Tatragas) was dried by bubbling through concentrated sulphuric acid. Used 
mixture was melted and the melt further kept at the constant temperature of $(680 \pm 2)^{\circ} \mathrm{C}$, controlled by a Pt-Pt10Rh thermocouple. After an immersion of metal sample, the polarization resistance and chronopotentiometric measurements were performed at approximately equal intervals. At polarization resistance measurements, the dependence $I=f(E)$ was measured in the potential range $\pm 200 \mathrm{mV}$ from the corrosion potential Ecor with polarization rate $0.001 \mathrm{~V} \cdot \mathrm{s}^{-1}$. At chronopotentiometric studies, the dependence $E=f(t)$ at constant current was measured from the open circuit potential (OCP) to the cathodic limit.

Surface of samples after corrosion was analysed by scanning electron microscope (Zeiss EVO 40, REM TESLA-BS 300, Slovakia) and X-ray microanalysis (EDX, probe EPMA JEOL JXA-840 A, Japan) that was, together with X-ray diffraction analysis (STOE stadi P, Bruker D8 Advance Super Speed, Germany), also used for the identification of corrosion products.

\section{Results and Discussion}

In Fig. 1, comparison of the time dependence of corrosion current density obtained during corrosion of tungsten in inert argon and oxidation air atmosphere is shown. As can be seen from figure, corrosion rate has (except of initial stage) the same trend in both types of atmospheres, although it is higher in the presence of air (oxygen) than in its absence. From the final stage of corrosion measurement it can be assumed that the corrosion rate will remain constant. Overall change of thickness of tungsten (calculated under assumption that the average number of electrons exchanged at the electrochemical process is 3 ) in this stage of corrosion

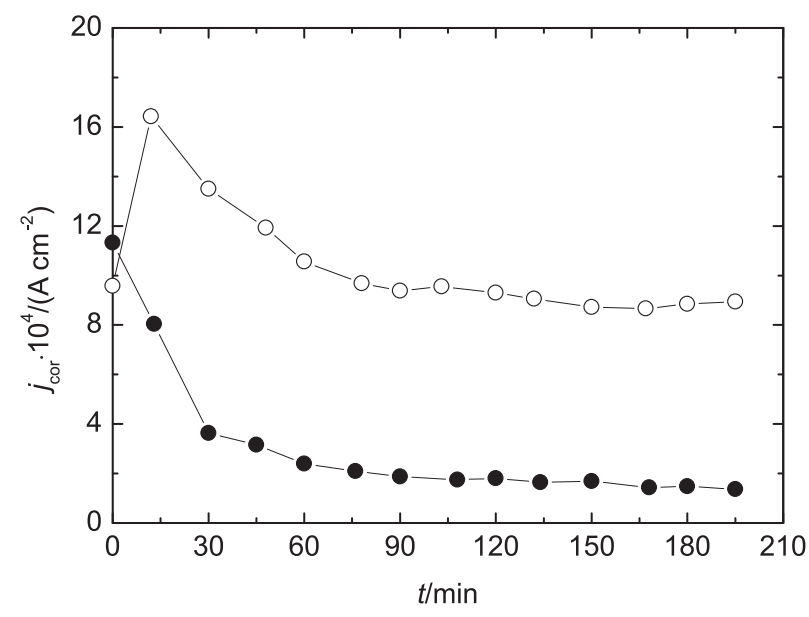

Fig. 1. Comparison of the corrosion current density obtained during corrosion of tungsten in argon atmosphere $(\bigcirc)$ and in air atmosphere $(\bigcirc)$. corresponds to the value of $2.9 \times 10^{-1} \mathrm{~cm} \cdot \mathrm{r}^{-1}$ and $10.9 \times 10^{-1} \mathrm{~cm} \cdot \mathrm{r}^{-1}$ in the absence and presence of air, respectively.

It can be concluded that presence of air significantly decreases corrosion resistance of tungsten. This decrease was not observed in the molten eutectic system LiF-NaF-KF (Ambrová and Danielik 2012), where corrosion resistance of tungsten in the absence of air was comparable with corrosion resistance of tungsten in the presence of air. This phenomenon was attributed to the formation of grains of corrosion products on tungsten surface during corrosion in the presence of air that partly inhibited corrosion process.

Corrosion attack of tungsten can be also seen in Fig. 2, where the surface of tungsten before and after corrosion in the absence and presence of air obtained by scanning electron microscope is shown. While the surface of tungsten has a uniform microstructure without any significant inhomogeneities before corrosion measurement (a typical texture is caused by the grinding of electrode surface), an occurrence of the areas with a new phase can be observed after corrosion measurement; the visible material contrast corresponds to the phase separation. After the corrosion measurement in the presence of air (oxygen), the surface becomes already uniformly crinkled, covered with a newly formed crystalline or granular layer.

Composition of the corrosion products present on the surface of tungsten and in the solidified melt after corrosion measurements is listed in Table 1. As follows from table, no corrosion products were identified in the solidified melt after corrosion measurement performed in the absence or presence of air. Based on the above given results and appearance of the solidified melts after corrosion measurements (melt after corrosion in the absence of air was coloured by corrosion products) it can be assumed that corrosion products formed in the presence of air are insoluble in the melt and remain on the metal surface, what partly inhibits corrosion process, while corrosion products formed in the absence of air are soluble in the melt (content below a detection limit of X-ray diffraction analysis). Observed decrease and stabilization of the corrosion rate (see Fig. 1) is most probably caused by saturation of the melt by corrosion products close to the metal surface and their slow diffusion into the bulk of the melt.

In Fig. 3, comparison of the time dependence of corrosion current density obtained during corrosion of nickel in inert argon and oxidation air atmosphere is shown. As can be seen from figure, corrosion rate has the same trend in both cases. However, after approx. $75 \mathrm{~min}$ it becomes significantly higher in 

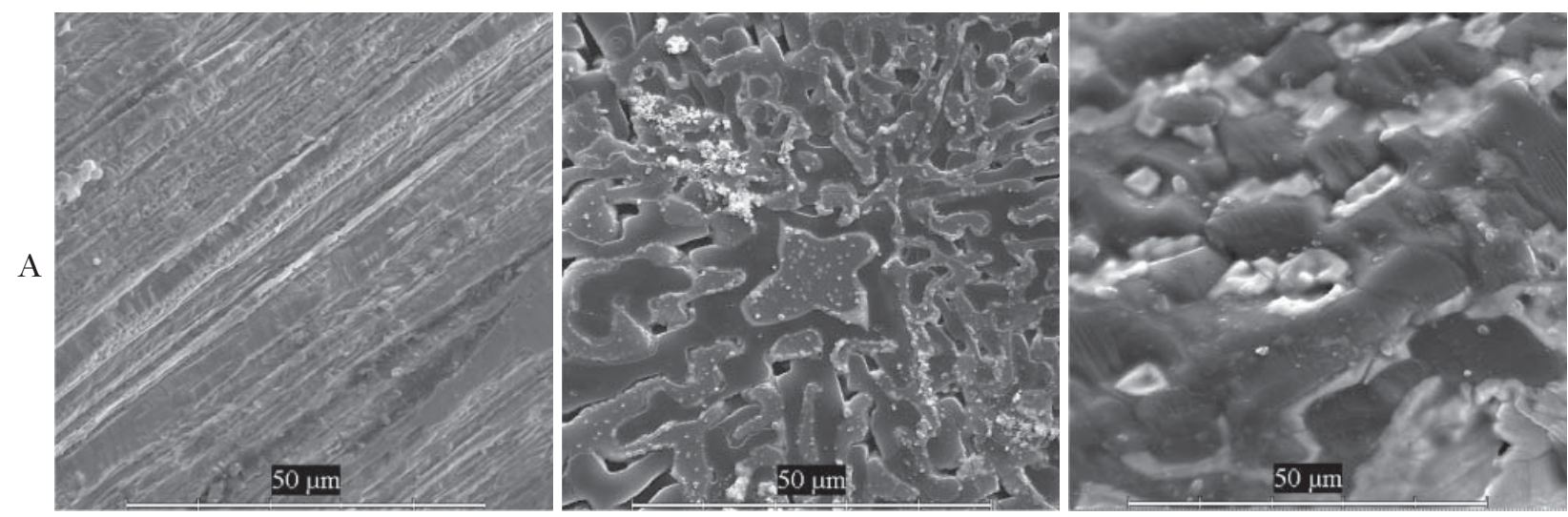

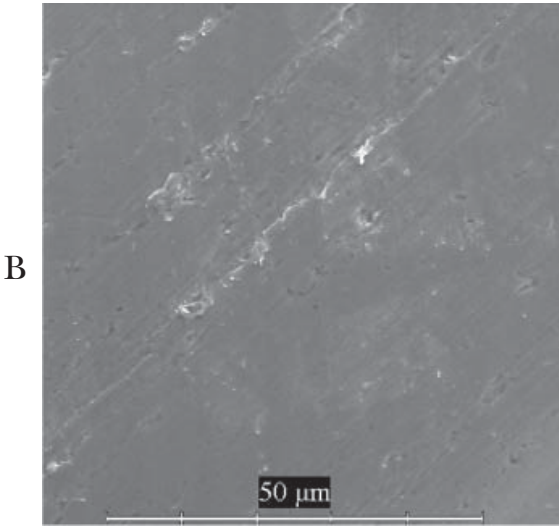

before corrosion

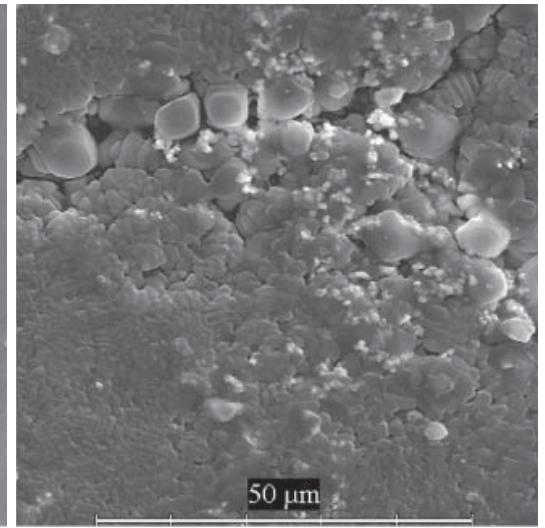

after corrosion in Ar atm

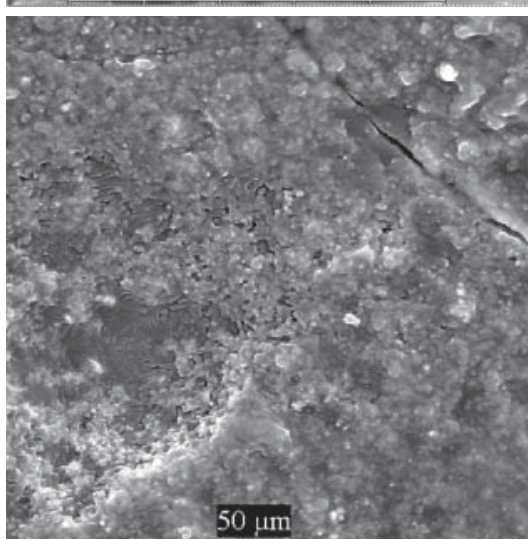

after corrosion in air atm

Fig. 2. Surface of tungsten (A) and nickel (B) before and after corrosion.

Tab. 1. Composition of the melt and surface of metal samples after corrosion.

\begin{tabular}{lcccc}
\hline \multirow{2}{*}{ Sample } & \multicolumn{2}{c}{ Composition of the melt } & \multicolumn{2}{c}{ Composition of the surface } \\
\cline { 2 - 5 } & Argon atm & Air atm & Argon atm & Air atm \\
\hline tungsten & $\mathrm{LiF}, \mathrm{NaF}$ & $\mathrm{LiF}, \mathrm{NaF}$ & $\mathrm{W}, \mathrm{LiF}, \mathrm{NaF}$ & W, probably $\mathrm{Na}_{3} \mathrm{WO}_{3} \mathrm{~F}_{3}, \mathrm{Na}_{2} \mathrm{~W}_{4} \mathrm{O}_{13}$ \\
\hline nickel & $\mathrm{LiF}, \mathrm{NaF}$ & $\mathrm{LiF}, \mathrm{NaF}$ & $\mathrm{Ni}, \mathrm{Li}_{2} \mathrm{NiF}_{4}$ & $\mathrm{Ni}_{4} \mathrm{LiNiO}_{2}, \mathrm{NiO}_{2} \mathrm{Ni}_{2} \mathrm{O}_{3}$ \\
\hline
\end{tabular}

the presence of air (oxygen) than in its absence. From the final stage of corrosion measurement it can be assumed that the corrosion rate will slightly decrease to the some constant value. Overall change of thickness of nickel (calculated under assumption that the average number of exchanged electrons during process is 2) in this stage of corrosion corresponds to the value of $13.8 \times 10^{-1} \mathrm{~cm} \cdot \mathrm{r}^{-1}$ and $31.9 \times 10^{-1} \mathrm{~cm} \cdot \mathrm{r}^{-1}$ in the absence and presence of air, respectively. It can be concluded that the presence of air (oxygen) in the molten eutectic LiF-NaF decreases corrosion resistance of nickel. Similar trend was also observed in the molten eutectic LiF-NaF-KF (Ambrová and Danielik 2012), although corrosion resistance of nickel measured in this system under argon atmosphere (i.e. in the absence of air) was higher probably due to faster saturation of the melt by corrosion products.

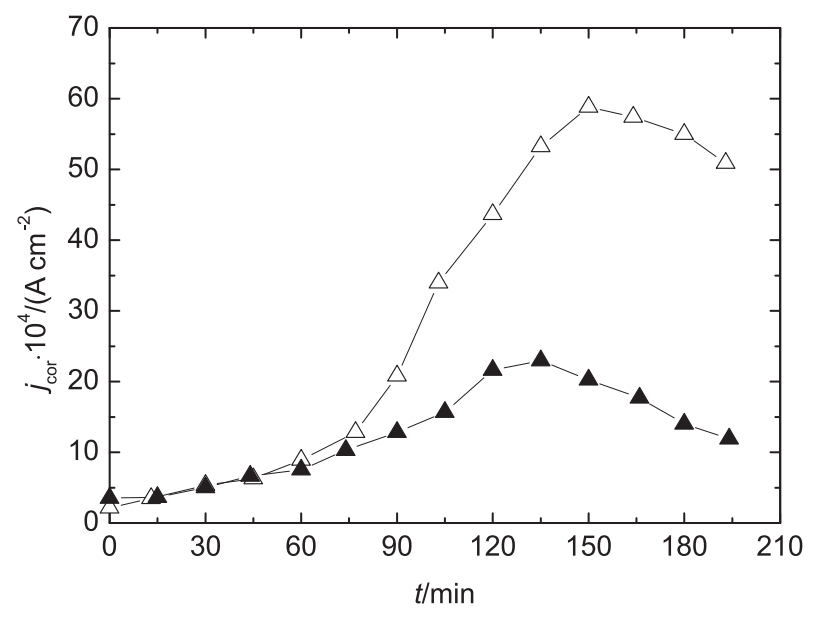

Fig. 3. Comparison of the corrosion current density obtained during corrosion of nickel in argon atmosphere $(\boldsymbol{\Delta})$ and in air atmosphere $(\triangle)$. 
In Fig. 2, surface of nickel before and after corrosion in the absence and presence of air obtained by scanning electron microscope is shown. While the surface of nickel has a uniform microstructure without any significant inhomogeneities before corrosion measurement (a typical texture is caused by the grinding of electrode surface), the formation of continuous, undulated layer with a characteristic segmentation to the block, that consist of the melt with a visible phase separation and gradual formation of new crystalline phases, can be observed after the corrosion measurement performed in the absence of air. During the corrosion measurements performed in the presence of air, the continuous, wrinkled layer with occurrence of the areas with phase unmixing and the seeds of crystalline phases was formed.

As follows from Table 1, the formation of lithiumnickelfluorid was detected on the electrode surface after corrosion measurement performed in the absence of air. Based on the above given results and appearance of the solidified melt after corrosion measurements in the absence of air (melt was coloured by corrosion products) it can be assumed that corrosion products are soluble in the melt, although no nickel compounds were found in the solidified melt (content below a detection limit of X-ray diffraction analysis). This supports corrosion process of nickel under argon atmosphere and partly explains its lower corrosion resistance in comparison with the molten system LiF-NaF-KF, where the concentration of corrosion products in the melt was higher, so the saturation of the melt in the vicinity of metal surface became earlier (Ambrová and Danielik 2012).

Compounds of nickel with oxygen detected on the electrode surface after corrosion measurement per-

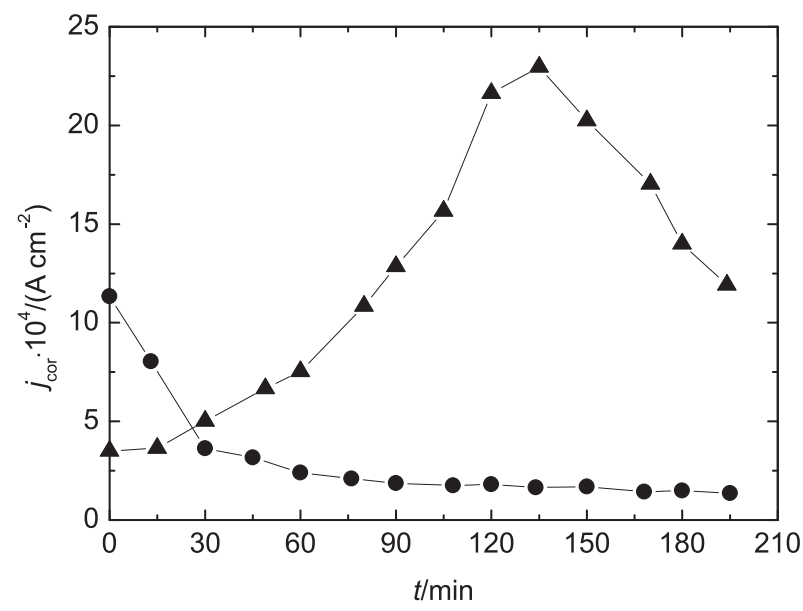

Fig. 4. Comparison of the corrosion current density obtained during corrosion of tungsten ( and nickel ( $\mathbf{\Lambda})$ in argon atmosphere. formed in the presence of air in the molten eutectic LiF-NaF supports passivation of nickel surface, similarly as is known from atmospheric corrosion. However, the formation of non-adhesive layers that were cracked from the metal surface caused, that no passivation of nickel surface occurred in the molten eutectic LiF-NaF-KF and that the corrosion rate was rapidly higher in this case (Ambrová and Danielik 2012).

Comparison of the time dependence of corrosion current density obtained during corrosion of tungsten and nickel in inert argon and oxidation air atmosphere, respectively, is shown in Figs. 4 and 5. As can be seen, corrosion rate of tungsten and nickel rather differs in both types of atmospheres. While it decreases to the constant value in the case of tungsten, it firstly increases and after approx. $150 \mathrm{~min}$ decreases in the case of nickel. It can be concluded that tungsten is more corrosion resistant material than nickel in the molten eutectic LiF-NaF.

This trend was not observed in the molten eutectic LiF-NaF-KF, where nickel was more corrosion resistant metal under inert argon atmosphere and tungsten under oxidation air atmosphere (Ambrová and Danielik 2012). Higher corrosion resistance of nickel in the absence of air was probably caused by fast saturation of the melt by corrosion products what inhibited the corrosion process in this system. In Fig. 6, comparison of the surface concentration of corrosion products determined by chronopotentiometry and digital simulation based on polarization resistance measurement is shown. Digital simulation was based on the approach presented in (Feldberg 1969), whereby the concentration of corrosion products on the metal surface (nickel) was calculated (diffusion coefficient $D=5 \times 10^{-5} \mathrm{~cm}^{2} \cdot \mathrm{s}^{-1}$ and number of exchanged elec-

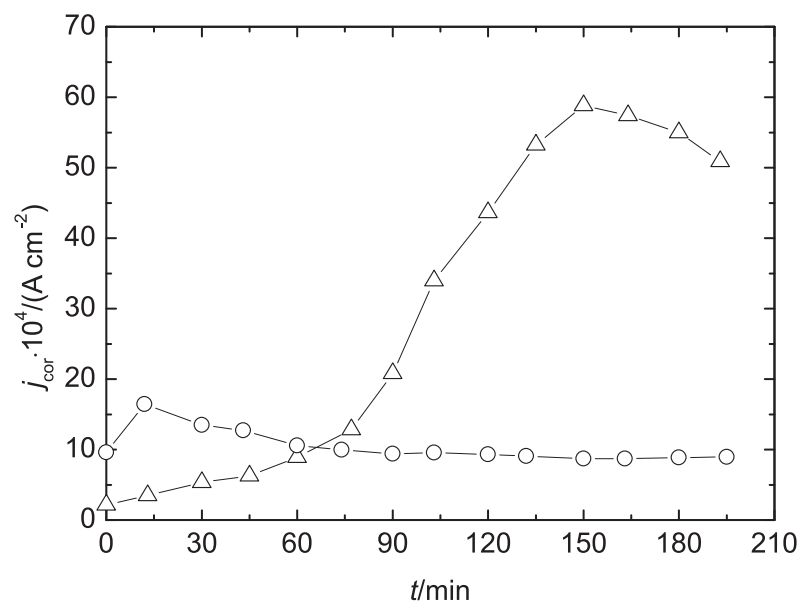

Fig. 5. Comparison of the corrosion current density obtained during corrosion of tungsten $(O)$ and nickel $(\triangle)$ in air atmosphere. 


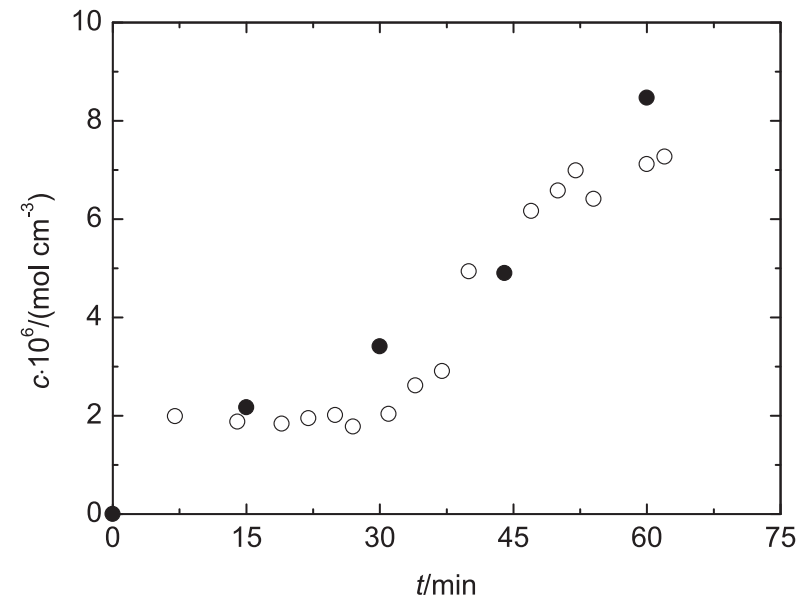

Fig. 6. Comparison of the surface concentrations of corrosion products obtained by chronopotentiometry $(\bigcirc)$ and by digital simulation based on polarization resistance measurement $(\mathbf{O})$.

trons $n=2$ were considered in simulation). As can be seen from figure, the results of both methods are in a good agreement. However, after $60 \mathrm{~min}$ of the corrosion measurement, concentration of corrosion products obtained by chronopotentiometry was not comparable with the results of polarization resistance measurements due to slow "dissolution" of the corrosion products formed on the electrode surface that was in this manner partly blocked. This assumption supports also increasing trend of the dependence $-j \tau^{1 / 2}$ on cathodic current density (Ambrová et al. 2006) that is shown in Fig. 7 and the absence of corrosion products in the solidified melt after the corrosion measurements.

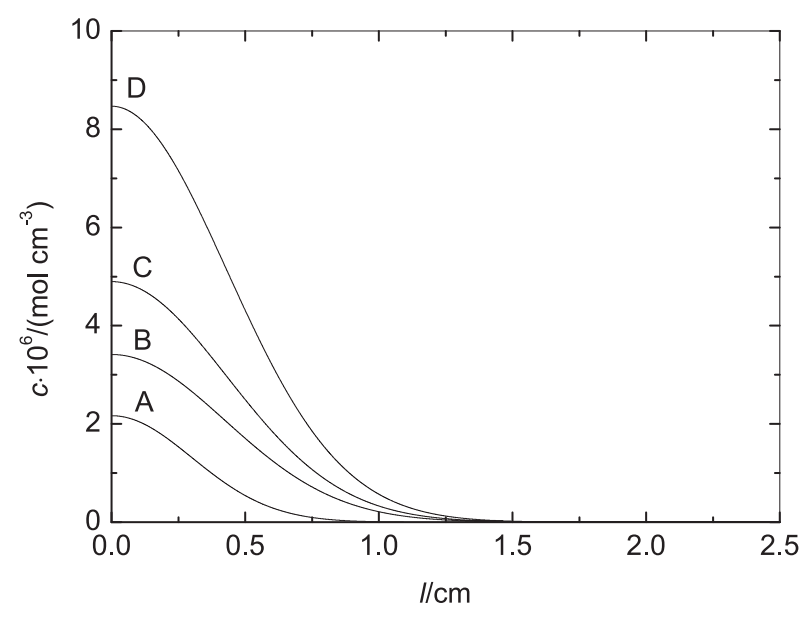

Fig. 8. Concentration profiles of corrosion products obtained by digital simulation at different stages of corrosion (15 min (A), $30 \mathrm{~min}$ (B), $44 \mathrm{~min}$ (C), $60 \mathrm{~min}(\mathrm{D}))$. Points plotted at zero distance (metal surface) represent concentrations obtained by chronopotentiometry.

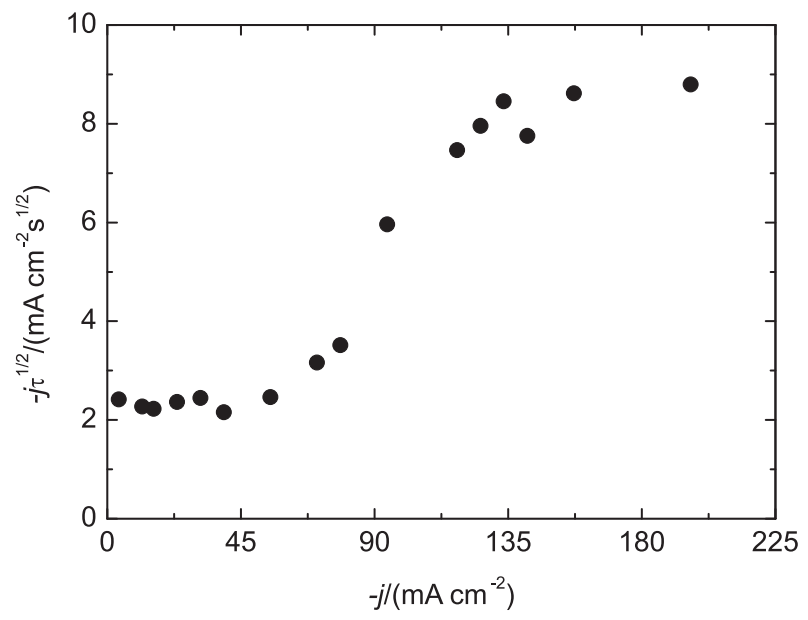

Fig. 7. Dependence of the term $-j \tau^{1 / 2}$ on cathodic current density obtained during chronopotentiometric measurements on nickel electrode.

From the time dependence of concentration of electroactive species measured by chronopotentiometry during the corrosion of nickel (see Fig. 6) it can be assumed that corrosion rate will slowly decrease with time. This is also in agreement with the measurement of polarization resistance. Similar results were obtained also in the molten eutectic system LiF-NaF-KF (Ambrová and Danielik 2012).

In Fig. 8, concentration profiles of corrosion products obtained by digital simulation at different stages of nickel corrosion are shown. As follows from figure, a saturation level of the corrosion products can be achieved in the vicinity of metal surface (matrix metal). Thus the soluble corrosion products can crystallize on the metal surface and in this manner partly inhibit the corrosion process. This is in agreement with the experimental results listed above.

\section{Conclusion}

It can be concluded that the presence of air (oxygen) in the investigated molten eutectic systems decreases corrosion resistance of tungsten and nickel that is negative mainly in term of safety during some failure.

When comparing tested metals, except of nickel exposed to the molten eutectic LiF-NaF-KF under argon atmosphere, tungsten is more corrosion resistant metal in both investigated molten eutectic systems and atmospheres, i.e. in the absence and presence of air (oxygen).

In term of investigated systems, the molten eutectic mixture $\mathrm{LiF}-\mathrm{NaF}$ is more suitable than molten eutectic mixture LiF-NaF-KF. KF increases probability of the presence of oxygen in the system (KF 
Tab. 2. Overall change of thickness $(\Delta d)$ evaluated for the final stage of corrosion in the molten eutectic systems LiF-NaF and LiF-NaF-KF (in parentheses).

\begin{tabular}{lcc}
\hline \multirow{2}{*}{ Sample } & \multicolumn{2}{c}{$\Delta \boldsymbol{d} \cdot \mathbf{1 0 ^ { 1 } / ( \mathbf { c m } \cdot \mathbf { r } ^ { - \mathbf { 1 } } )}$} \\
\cline { 2 - 3 } & Inert argon atmosphere & Oxidation air atmosphere \\
\hline tungsten & $2.9(8.1)$ & $10.9(9.4)$ \\
nickel & $13.8(2.5)$ & $31.9(144.3)$ \\
\hline
\end{tabular}

je highly hygroscopic) which can decrease the corrosion resistance of metals.

As follows from Table 2, rather high values of the overall change of thickness $(\Delta d)$, evaluated for the final stage of corrosion measurements, indicate that any of the investigated metals cannot be itself used neither in the molten eutectic mixture LiF-NaF nor LiF-NaF-KF.

Based on the analysis of corrosion products it can be assumed that nickel anodically oxidizes to $\mathrm{Ni}^{2+}$ ions during corrosion process. This assumption was also confirmed by the independent chronopotentiometric and polarization resistance measurements, where the results of both methods were in agreement when considering the two exchanged electrons.

\section{Acknowledgement}

This work was supported by courtesy of the Slovak Grant Agency (VEGA 1/0588/11).

\section{References}

Ambrová M, Danielik V (2012) Acta Chimica Slovaca 5: $5-9$.

Ambrová M, Danielik V, Fellner P, Thonstad J (2006) Electrochimica Acta 51: 5825-5828.

Feldberg SW (1969) Electroanalytical Chemistry 3: 199-296. 